\title{
Performance of Orbitrap Mass Analyzer at Various Space Charge and Non-Ideal Field Conditions: Simulation Approach
}

\author{
Andriy Kharchenko, ${ }^{1}$ Gleb Vladimirov, ${ }^{2,3}$ Ron M. A. Heeren, ${ }^{1}$ Eugene N. Nikolaev ${ }^{2,3,4}$ \\ ${ }^{1}$ FOM Institute for Atomic and Molecular Physics (AMOLF), Amsterdam, The Netherlands \\ ${ }^{2}$ Institute for Energy Problems of Chemical Physics, Russian Academy of Sciences, Moscow, Russia \\ ${ }^{3}$ Emanuel Institute of Biochemical Physics of Russian Academy of Sciences, Moscow, Russia \\ ${ }^{4}$ Institute of Biomedical Chemistry of Russian Academy of Medical Sciences, Moscow, Russia
}

\begin{abstract}
The orbital trap mass analyzer provides a number of unique analytical features along with inevitable limitations as an electrostatic instrument operating in high space charge regimes resulting in systematic measured frequency errors as an effect of stored ion clouds on the trap field and each other effect of non-ideal machining the trap electrodes, effect of injection slot, effect of real versus theoretical trap dimensions, etc. This paper deals with determining the influence of the space charge effect and imperfection of the electrostatic field on the motion of ion ensembles in the orbital trap. We examine effects of theoretically modeled non-harmonicity of the electrostatic potential and the number of confined ions on stability of coherent ion motion in the trap that determines the frequency shifts of axial ion oscillation. Three different Orbitrap geometries were considered: geometry close to preproduction Orbitrap, close to standard Orbitrap, close to high field Orbitrap. Frequency shifts for $m / z=500$ and for charge state +23 of cytochrome $\mathrm{c}$ isotopic cluster particles with $10^{4}-6^{*} 10^{6}$ elemental charges in the trap were considered. Refined spectra were calculated using the filter diagonalization method proposed by Mandelshtam et al. and applied to mass spectrometry by O'Connor and Aizikov.
\end{abstract}

Key words: Orbitrap, Frequency Shifts, Fourier Transform, FDM, Inharmonic Oscillations, Trap potential Expansions, Inharmonic Potential, Simulation

\section{Introduction}

$\mathrm{T}$ he use of mass spectrometry in analysis of biological samples makes high demands to resolution (over $10^{6}$ ) and accuracy (above ppm) of mass measurement. For a long long time, these demands were only met by the Fourier transform ion cyclotron resonance mass spectrometry (FTICR) [1]. However, while providing for unsurpassed performance, especially with recently introduced dynamically harmonized cells [2,3], this method is not free of some drawbacks like the need for high magnetic field cryomagnets

Correspondence to: Ron M. A. Heeren; e-mail: heeren@amolf.nl, Eugene Nikolaev; e-mail: ennikolaev@rambler.ru incurring significant usage expenses. For this reason, there is growing interest in another design of Fourier transformbased spectrometers - the recently invented orbital ion trap having performance comparable to FT-ICR instruments, which have ion traps without dynamic harmonization. Instruments based on the orbital ion trap have gained recognition owing to work by Makarov who had implemented the principle proposed by Kingdon [4]. Currently there is a considerable amount of understanding of limitations of spectrometry based on the principle of orbital charged particle trapping and ways of improving its analytical performance. Trap field inharmonicities, geometric defects, and the space-charge effect are known to be major factors compromising the resolution and accuracy of 
Orbitrap-based mass spectrometry [5-7]. Particularly, ion loss behind the process of transient decay is caused by collisions with background gas, trapping field imperfections, and the space-charge effect. Sensitivity to collisions (uniform in $\mathrm{m} / \mathrm{z}$ for the Orbitrap) requires a ultra-high vacuum especially for "heavy" compounds. In this paper, we address the following factors: (1) space-charge effect, which causes loss of ion packet coherence and affects ions kinetic energy in the axial direction, (2) unintentional defects of trap electrode geometry caused by the injection slot and inaccurate machining, and (3) inevitable trapping potential inharmonicities as a result of the trap"s finite size.

\section{Experimental}

\section{Motion in the Orbital Trap}

The Orbitrap's operation is based on ions motion in the electrostatic field induced by a potential [8]:

$$
\varphi_{t r}(r, z)=\frac{k}{2}\left(z^{2}-\frac{r^{2}}{2}\right)+\frac{k}{2} R_{m}^{2} \ln \frac{r}{R_{m}}+C
$$

where $k, C$ are constants, $R_{m}$ - characteristic radius, $k$ - field curvature; $r^{2}=x^{2}+y^{2} ; x, y, z$ - Cartesian coordinates oriented the way that $Z$ is the symmetry axis. Such a field can be created by a system of two coaxial, radially symmetric electrodes whose geometry can be described in the form of function $Z(r)$ :

$$
z(r)_{1,2}=\sqrt{\frac{r^{2}}{2}-\frac{R_{1,2}^{2}}{2}+R_{m}^{2} \ln \left(\frac{R_{1,2}}{r}\right)}
$$

where $R_{1}, R_{2}$ are geometrical parameters. The motion of an individual ion having a near-elliptical trajectory can be represented by three uncoupled types of motion: rotation around the central electrode located on axis $Z$, oscillations towards $Z$, and oscillations along $Z[8,9]$. Thus the latter oscillation

$$
m \ddot{Z}=-q E_{z}=-q \frac{\partial \varphi}{\partial Z}=-q k Z
$$

is useful for mass analysis, and its frequency can be expressed as

$$
\omega=\sqrt{\frac{q}{m} k}
$$

For a given radius of the circular orbit $R$, the frequencies of radial oscillations and rotation are, respectively:

$$
\omega_{r}=\omega \sqrt{\frac{R_{m}^{2}}{R^{2}}-2}
$$

and

$$
\omega_{\varphi}=\omega \sqrt{\frac{1}{2}\left(\frac{R_{m}^{2}}{R^{2}}-1\right)}
$$

\section{Numerical Simulation}

A popular framework to explore mass, resolution, and sensitivity in electromagnetic ion traps is based on observing the space charge effect addressed by the particle in cell approach [10], which is in turn based on solving the Poisson equation at each time step of particle motion integration. We followed this approach and combined the theoretically calculated field of the (unperturbed) orbital trap with the field of confined charged particles by the principle of superposition. The simulation presets included a specific time step to provide 100 revolutions around the central electrode, similar to ICR simulations where the minimum cyclotron period contains 100 steps. We also checked different grid coarseness and found that $64 \times 64$ in the $\mathrm{XY}$ plane provide the optimal performance without loss of precision. During the simulations, we found that the initial conditions of ions resulted from the ion injection process significantly affects their later evolution. The size of the simulation cube was chosen so that the outer electrode would fit into it at $Z \approx 0$ and all the ions would be confined with the cube. Secondly, the cube's side length $L$ was tuned the way that it would be expressed in an integer number of length units of 0.5 in. $=1.27 \mathrm{~cm}$ used in our simulation program, therefore $L=3.0$.

\section{Determining Basic Geometric Parameters}

In order to obtain comparable results, we need to define the principal geometric parameters of the trap. We resort to open sources and mostly theory only since those parameters are proprietary manufacturing information. Thus, a specific Orbitrap geometry can be determined via the electric field curvature $k$ and the effective radius $R_{m}$. We know [11] that for the standard Orbitrap $R_{1}=6 \mathrm{~mm}, R_{2}=15 \mathrm{~mm}, R_{m} \approx R_{2} \sqrt{2}$, and $U=3500 \mathrm{~V}$; for the high-field Orbitrap $R_{1}=9 \mathrm{~mm}, R_{2}=$ $15 \mathrm{~mm}, R_{m} \approx R_{2} \sqrt{2}$, and $U_{r}=5000 \mathrm{~V}$; for the pre-industrial design [12] $R_{1}=7.393 \mathrm{~mm}, R_{2}=19.96 \mathrm{~mm}, R_{m}=$ $32.388 \mathrm{~mm}$, and that $\mathrm{m} / \mathrm{z} 280.169$ corresponds to $\omega=246762.0 \mathrm{~Hz}$. Thus, given knowledge of the spindle's $R_{1}$, $R_{2}$, and $U$ we can estimate $k$ and $\omega$, or given $R_{1}, R_{2}, R_{m}$, and $\omega$ we can get $U$. With respect to the fact that the outer electrode is grounded and some voltage $U$ is applied to the spindle, we have: $\varphi\left(R_{2}\right)=0$ and $\varphi\left(R_{1}\right)=U$. Then by expressing $Z$ via $R_{1}$ and $R_{2}$ using (2) we finally have:

$$
k=\frac{2 U}{R_{m}^{2} \ln \frac{R_{2}}{R_{1}}-\frac{1}{2}\left(R_{2}^{2}-R_{1}^{2}\right)}
$$

Using this expression, we can connect the frequency with dimensional and voltage parameters of various Orbitrap versions (see Table 1).

\section{Ion Injection}

During injection, voltage is applied to the central electrode in order to attract ions moving in the proximity of the electrode. 
Table 1. Orbitrap Geometries

\begin{tabular}{lcccccc}
\hline Geometry & $R_{1}[\mathrm{~mm}]$ & $R_{2}[\mathrm{~mm}]$ & $R_{m}[\mathrm{~mm}]$ & $U[\mathrm{~V}]$ & $f(500 \mathrm{Da})$ & $f_{\varphi}(500 \mathrm{Da})$ \\
\hline Pre-serial & 7.393 & 19.96 & 32.388 & 3000 & $1.8 \cdot 10^{5}$ & $3.8 \cdot 10^{5}$ \\
Standard & 6 & 15 & 22 & 3500 & $3.1 \cdot 10^{5}$ & $4.8 \cdot 10^{5}$ \\
High-field & 9 & 15 & 22 & 5000 & $5.2 \cdot 10^{5}$ \\
\hline
\end{tabular}

From the literature [12], we know that the axial amplitude of ion oscillations is estimated to to be $7 \pm 1.5 \mathrm{~mm}$, while trajectory diameters at the spindle equator lie within the range 24-30 $\mathrm{mm}$; therefore, the injection radius should be $13.5 \mathrm{~mm}$ at $Z=7 \mathrm{~mm}$. The initial cloud distribution was a uniform sphere having a radius of $0.12 \mathrm{~mm}$ and the initial velocity vector should be preset to mimic injection in a real trap.

The motion pattern significantly depends on these initial conditions. From [11], we know that the real injection system is designed the way that all the ions have a rotation speed $V_{\phi}$, which can be expressed via Equation 6 as

$$
\frac{m / z V_{\varphi}^{2}}{2 e}=\frac{k}{4}\left(R_{m}^{2}-R^{2}\right)
$$

Also based on that work, the injection system is known to have the asymptotic radius of rotation around the inner electrode in the interval $R=9-10 \mathrm{~mm}$ for the standard geometry and 10-10.5 $\mathrm{mm}$ for the high-field geometry. In a real instrument injection, the central electrode is set to some voltage when the ions are in its proximity. We simulated this process via the following set-up: at the initial moment the central electrode has some voltage applied, the ion cloud is located at some radius corresponding to the free orbital motion, and all the ions have the corresponding initial velocity.

The initial velocity and coordinates of particles are critical for the subsequent simulation so we experimentally pre-set them following the lines of tuning a real instrument to some extent. The ions in the cloud were initialized to have the same velocity at the direction of rotation and to be uniformly distributed inside a sphere of radius $r=0.12 \mathrm{~mm}$ having center coordinates $(R, Z)=(9,6) \mathrm{mm}$ ( standard geometry). In general, if the particle velocity in $X Y$-plane is different from the value given in Equation (6) then the revolution trajectory around the spindle will tend to be elliptical rather than circular. Accordingly, we experimentally optimized the initial velocity to minimize ellipticity of the asymptotic orbit. Figure 1 presents the simulation layout. Figure 2 presents cloud evolution at various initial conditions of injection. As expected, varying the initial velocity results in different trajectory ellipticity (area of ion cloud cross-section), particularly, the initial energy $1300 \mathrm{eV}$ results in greater ellipticity than $1600 \mathrm{eV}$. Injection parameters for various Orbitrap geometries used in this paper are presented in Table 2. This is in accordance with one of the results by $\mathrm{Hu}$ et al. [13] that the ellipticity of trajectory will depend on "energy match" of the tangential velocity and the radial potential.

\section{Simulating the Transient Generation}

The Orbitrap simulation not only calculates trajectories of ions in the trap, but also the time domain signal induced on the detection electrodes. The problem of accurate calculation of the current created in an arbitrary shaped electrode can be solved by allocating a number of virtual charges on the electrode surface. Then the detected signal is the current of charges induced between the halves of the outer electrode:

$$
I(t)=\frac{d Q_{k}}{d t}
$$
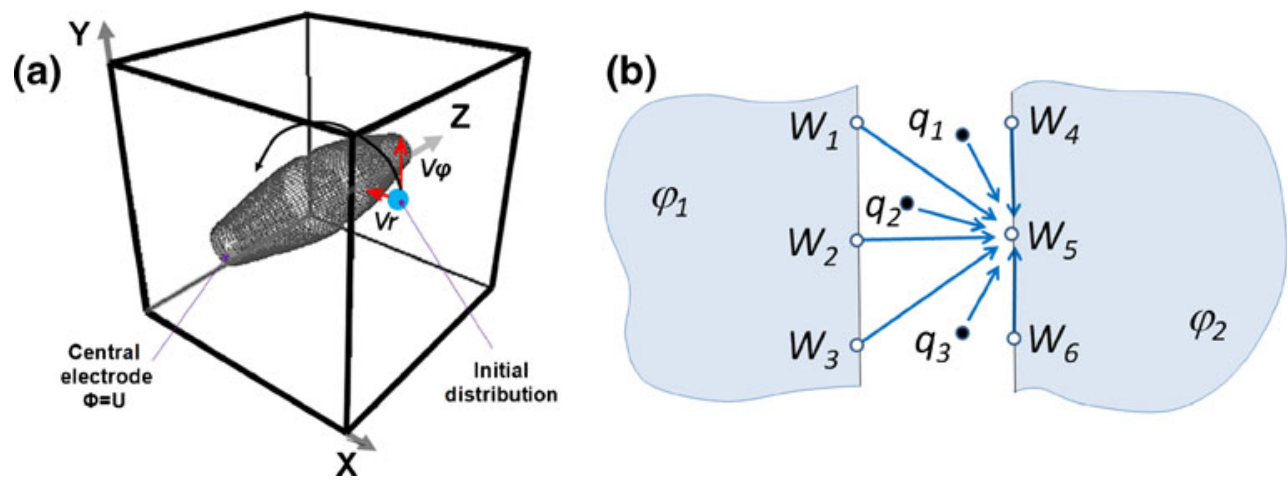

Figure 1. Schematic view of the numerical experiments. (a) Charged particles of spatial density $\rho$ are propagated driven by electric forces in the potential $\varphi_{t r}(r, z)=\frac{k}{2}\left(z^{2}-\frac{r^{2}}{2}\right)+\frac{k}{2} R_{m}^{2} \ln \frac{r}{R_{m}}+C$ between the inner and outer electrodes calculated via $\nabla^{2} \varphi=-\rho / \varepsilon_{0}$ in the grid $64 \times 64 \times 64$ elements. (b) Metal electrodes in a homogeneous dielectric. Ions are filled circles and induced image charges are empty circles located on the conductor surfaces. Arrows show the contributions that have to be summed for one image charge 


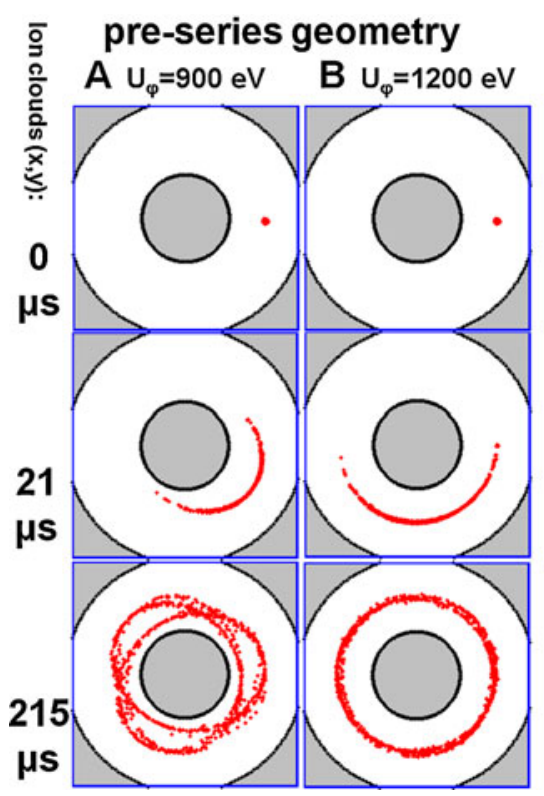

standard geometry

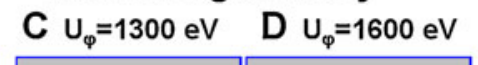

high-field geometry

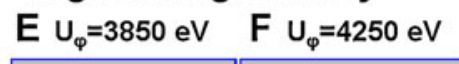

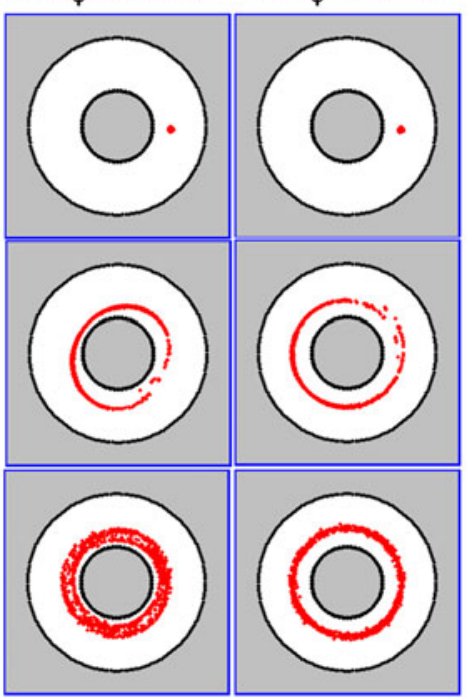

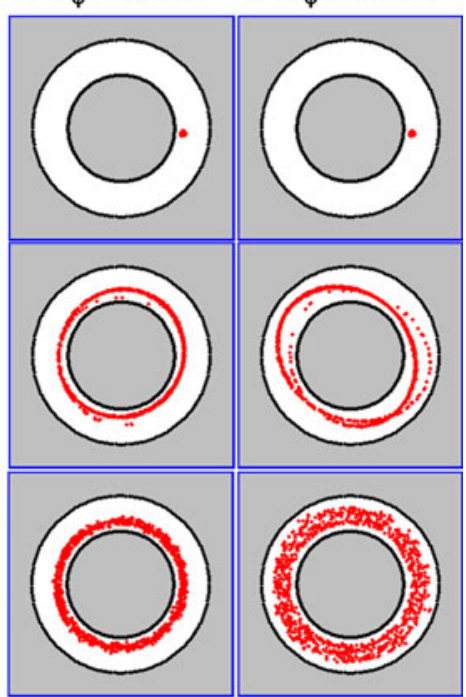

Figure 2. Motion pattern significantly depends on initial position and velocity vector of the ions in the cloud. In this figure, dynamics of $10^{3}$ ions $m / z=500, z=1 \mathrm{e}$ at various initial values of $V_{\varphi}$ in each Orbitrap geometry. Electrodes are grey, ions are red

induced by the image charge $Q_{k}$ of $i$-th ion in the outer split electrode by the ions' axial motion with respect to the surface charge polarization. Charge $Q_{k}$ is a sum of surface charges $W_{i}$ at discrete points of an electrode's surface

$$
Q_{k}=\sum W_{i}
$$

Such a discrete point charge will create a potential

$$
\varphi_{k}=\frac{W_{i}}{4 \pi \varepsilon r_{i}},
$$

where $\varepsilon$ is the electric permittivity, and we need to find the polarization of $W_{i}$ maintaining the equipotential electrode surfaces by adjusting the charges of those charges at discrete points. Thus, the discrete charges are uniformly distributed over each electrode surface to add up all potential contribution from each charge location and individual ions (see Figure 1).

If $W_{i}$ is the charge at the $i$-th discrete surface point and $r_{i j}$ is the distance between location $i$ and $j, q_{k}$ is the charge of $k$-th ion, and $d_{i j}$ is the distance between ion location $i$ and discrete point $j$, we can express potential $\varphi_{k}$ at location $h$ as

$$
\frac{W_{1}}{r_{h 1}}+\frac{W_{2}}{r_{h 2}}+\ldots+\frac{W_{n}}{r_{h n}}+\frac{q_{1}}{d_{h 1}}+\frac{q_{2}}{d_{h 2}}+\ldots+\frac{q_{m}}{d_{h m}}=4 \pi \varepsilon \varphi_{k}
$$

Table 2. Paramters of Injection for Each of the Orbitrap Geometries

\begin{tabular}{lccr}
\hline Geometry & $R[\mathrm{~mm}]$ & $Z[\mathrm{~mm}]$ & $U_{\varphi}[\mathrm{eV}]$ \\
\hline Pre-serial & 13.5 & 7 & 900,1200 \\
Standard & 9 & 6 & 1300,1600 \\
High-field & 10 & 6 & 3850,4250 \\
\hline
\end{tabular}

which for all the charges will result in a system

$\left(\begin{array}{cccc}0 & \frac{1}{r_{12}} & \ldots & \frac{1}{r_{1 n}} \\ \frac{1}{r_{21}} & 0 & \ldots & \frac{1}{r_{2 n}} \\ \ldots & & & \\ \frac{1}{r_{n 1}} & \frac{1}{r_{n 2}} & \ldots & 0\end{array}\right)\left(\begin{array}{c}W_{1} \\ W_{2} \\ \ldots \\ W_{n}\end{array}\right)=\left(\begin{array}{c}4 \pi \varepsilon \varphi_{1}-\frac{q_{1}}{d_{11}}-\frac{q_{2}}{d_{12}}-\ldots-\frac{q_{m}}{d_{1 m}} \\ 4 \pi \varepsilon \varphi_{2}-\frac{q_{1}}{d_{21}}-\frac{q_{2}}{d_{22}}-\ldots-\frac{q_{m}}{d_{2 m}} \\ \ldots \\ 4 \pi \varepsilon \varphi_{n}-\frac{q_{1}}{d_{n 1}}-\frac{q_{2}}{d_{n 2}}-\ldots-\frac{q_{m}}{d_{n m}}\end{array}\right)$

$\varphi_{k}$ is the potential of an electrode and can have one of two values, $\varphi_{1}$ or $\varphi_{2}$. The sum of surface charges is known to be 0 and the sum of potentials of the detection electrodes where these charges are located is also 0 . As a result we have a system of $n+2$ eqs for $n+2$ unknowns, which determines values of the charges induced on each electrode. System 13 was numerically solved via pseudo-inversion.

\section{Ion Cloud-Cloud Collision}

Collision of ion clouds with each other is a significant factor determining the dynamic range of the orbital trap resulting in a quick loss of close oscillation phases of ions. Unlike in FTICR mass analyzers, where frequencies of revolution are measured, the orbital trap is based on measuring frequencies of oscillations, which determines a significant difference in the spatial pattern of ion motion; in particular, ion cloud trajectories cross twice per period in the orbital trap, while in ICR traps it happens at a frequency equal to the difference of the clouds' cyclotron frequencies. Our simulations show that collisions of ion clouds with each other lead to the quick loss of phase in clouds of same $\mathrm{m} / \mathrm{z}$ when their population reaches $4 \times 10^{5}$ (Figure 3a). Simulation was performed for 2 clouds and for an isotope cluster of cytochrome $c$ protein, charge state +23 . 


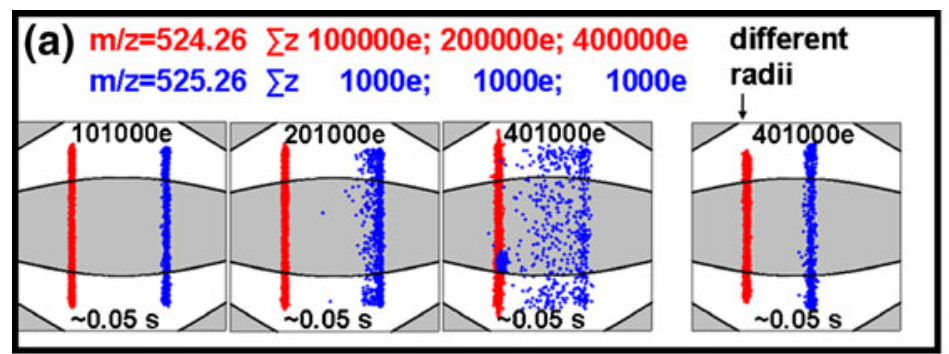

Ion clouds $(\mathrm{z}, \mathrm{y})$ :
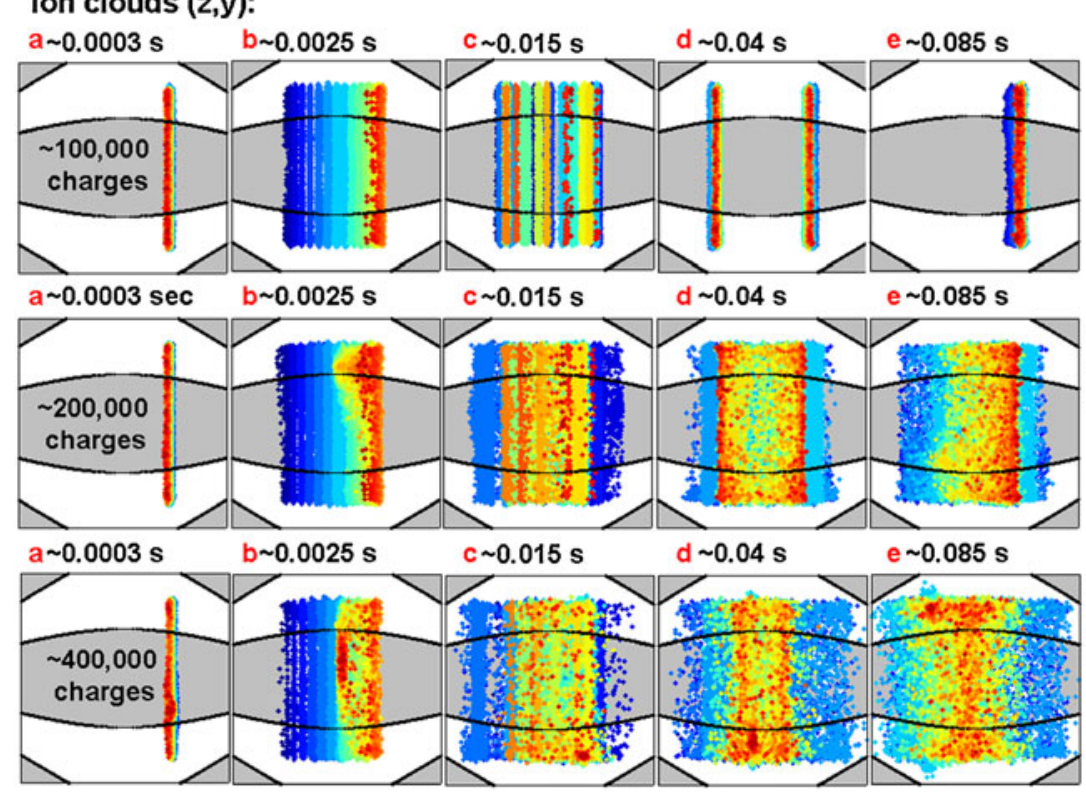

$511.5 \mathrm{~m} / \mathrm{z} 512.4$ (b) Time-Domain signal for 100,000 Charges( $0.1 \mathrm{~s}$ ):

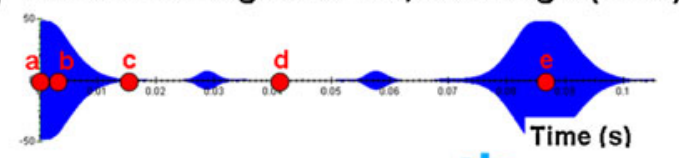

Isotope cluster Cytochrome c

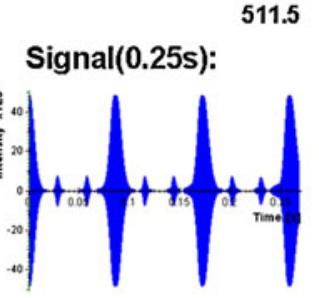

Frequencies:
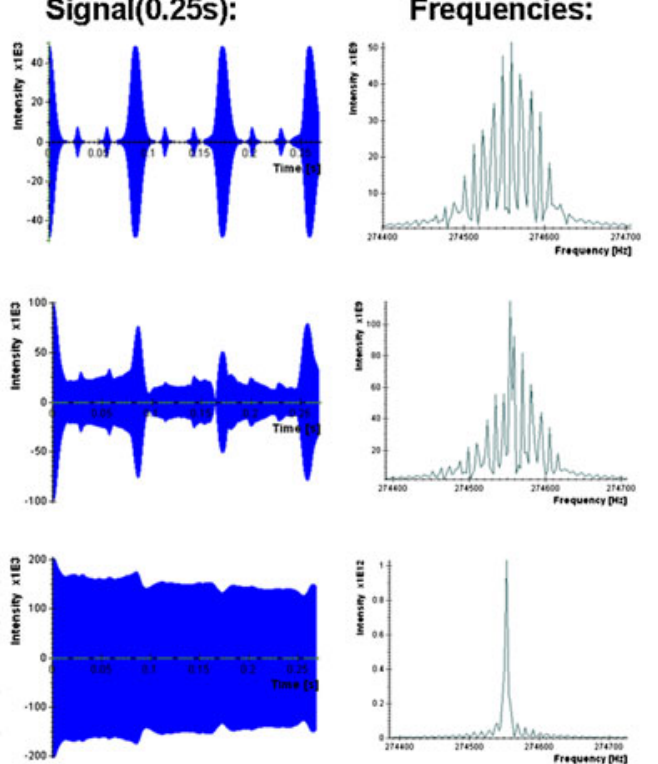

Figure 3. Evolution of ion cloud collision of two calibration masses and an isotope cluster of protein cytochrome $c$ (charge state +23 ) in an orbital trap mass analyzer $\left(U=3500 \mathrm{~V}, R_{1}=6 \mathrm{~mm}\right.$, and $R_{2}=22 \mathrm{~mm}$ ), central $1.27 \mathrm{~cm}$ fragment displayed. (a) Two calibration masses. (b) Cytochrome $c$ (from $\sim 10^{5}$ e to $\sim 4 * 10^{5} \mathrm{e}$ )

As a more practical example consider a spectrum of isotope cluster cytochrome $c$ of $10^{5}$ charges given charge state $Z=23, m \approx 512, d m \approx 0.043$, detection time $0.25 \mathrm{~s}$, 100 time steps per minimum frequency period of evolution around the central electrode. The mass list was produced using PNNL's molecular weight calculator [14]. The Fourier spectrum and the signal transient of $10^{5}$ ions of this isotope cluster are presented in Figure 3b. Visual analysis of the cloud evolution revealed that these changes of frequency occur during one cloud's passing through/by another one. Cloud destruction and loss of phase of particles occurs with the increase of the number of charges in an ion cluster. Broadening of the beats and a decrease in the number of spectral peaks can also be observed in this figure.

Concerning possible comparison with real commercial instruments, the authors were considering not a specific Thermo-manufactured device but a generic one due to not having access to the proprietary data of the instrument's dimensions and the duration and shape of the signal applied for ion capturing, as well as the scatter in time they arrive at the central electrode. Particularly, if the entry times of ions of different $\mathrm{m} / \mathrm{z}$ vary enough, they arrive at different orbits, hence, the effect of peak coalescence is not observed (see the last case in Figure 3a).

\section{Deviation from the Ideal Trapping Fields and Resolution}

In practice, to create an Orbitrap potential exactly as in (1) requires electrodes of geometry described by (2) and to have the infinite length by $Z$, which never takes place. In a real instrument, the electrode has a finite $Z$ length and, therefore, the resulting potential will deviate from (1) by some amount.

An ion trap has a rotational $(Z-)$ symmetry, therefore its field can be expanded by a series of spherical or cylindrical harmonics. For the case of electrostatic field in the FT-ICR trap, such an expansion by cylindrical harmonics was done in [15]. However, since term $\frac{k}{2}\left(z^{2}-\frac{r^{2}}{2}\right)$ of the ideal Orbitrap potential [1] is a spherical harmonic expansion term, we will stick to using that expansion further:

The Maxwell equation for the electrostatic field gives $\nabla \cdot E=\rho / \varepsilon_{0}$ and $\nabla \cdot E=0$ The latter is equivalent to the statement that $E$ is the gradient of the scalar potential $\varphi$ and $E=-\nabla \varphi$. Therefore the electrostatic field could be described 
by the Poisson equation $\nabla^{2} \varphi=-\rho / \varepsilon_{0}$. In regions of space lacking charge density, the scalar potential satisfies the Laplace equation $\nabla^{2} \varphi=0$. The general solution for boundary-value problems of the Laplace equation in spherical coordinates can be written in terms of spherical harmonics and powers of $r$ [16]:

$$
\varphi(r, \theta, \phi)=\sum_{l=0}^{\infty} \sum_{m=-l}^{l}\left[A_{l m} r^{l}+B_{l m} r^{-(l+1)}\right] Y_{l m}(\theta, \phi)
$$

where $Y_{l m}$ is a spherical harmonic. Note that $\frac{k}{2}\left(z^{2}-\frac{r^{2}}{2}\right)$ is a term of series expansion $A_{20} r^{2} Y_{20}$. For simplicity, we approximate imperfections of the field by series expansion $A_{30} r^{3} Y_{30}$ and $A_{40} r^{4} Y_{40}$. Therefore, we represent an electrostatic potential by:

$$
\begin{aligned}
\varphi_{t r}(r, z)= & \frac{k}{2}\left(z^{2}-\frac{r^{2}}{2}\right)+\frac{k}{2} R_{m}^{2} \ln \frac{r}{R_{m}}+\frac{k A_{30}}{L} \\
& \times\left(2 z^{3}-3 z r^{2}\right)+\frac{k A_{40}}{L^{2}} \\
& \times\left(8 z^{4}-24 z^{2} r^{2}+3 r^{4}\right)+C
\end{aligned}
$$

where $L$ - simulation region length, $C$ - constant, $A_{30}$, and $A_{40}$ are dimensionless electric field inharmonicity constants.

Expression (15) was obtained from (1) by adding term $A_{30} r^{3} Y_{30}$ (non-symmetric by $Z$ ) and $A_{40} r^{4} Y_{40}$ (symmetric by $Z$ ).
The idea behind this selection of terms was to provide the simplest approximation for exploring the behavior of ion clouds at the inevitable non-harmonicity of the trapping potential. As for terms of type $A_{l m} r^{l} Y_{l m}$ and $B_{l m} r^{-(l+1)} Y_{l m}$, if $m$ is non-zero, then the field is not rotation-symmetric while in our case we can assume that the trap field has this property and its orthogonal components should have the $Z$-axis rotational symmetry: $A_{11} r Y_{11}=A_{11} x, B_{11} r^{-2} Y_{11}=B_{11} y, A_{21} r Y_{21}=A_{21} z x, B_{21} r$ $Y_{21}=B_{21} z y$, and so forth. Therefore terms of this kind were not considered in deriving formula (15). As for terms $B_{l 0} r^{(l+1)} Y_{l 0}$, we can see that for $B_{10} r^{-2} Y_{10}=$ $B_{10} z /\left(x^{2}+y^{2}+z^{2}\right)^{(3 / 2)}, B_{20} r^{-3} Y_{20}=B_{20}\left(2 z^{2}-x^{2}-y^{2}\right) /$ $\left(x^{2}+y^{2}+z^{2}\right)^{(5 / 2)}$ and so on, their contribution vanishes at $z \rightarrow \infty$, while the goal of introducing additional terms was decreasing the field with the increase of $|Z|$ - the additional terms should have cancel the contribution of term $k / 2\left(z^{2}-x^{2} / 2-y^{2} / 2\right)$. Therefore, the terms of this kind were omitted. Regarding using a bigger number of terms of type $A_{l 0} r^{l} Y_{l 0}$, we tended to minimize the number of terms making them sufficient for a qualitative analysis of ion cloud behavior.

Presence of terms $\frac{A_{30} k}{L} 3 z r^{2}$ and $\frac{A_{40} k}{L^{2}} 24 z^{2} r^{2}$ in the trapping electrostatic potential also causes the $Z$-motion and the radial motion to stop being independent which in turn causes the $Z$-oscillations to depend on the trajectories of ion revolution around the central electrode. Since ion trajectories are scattered to some extent by ellipticity, this in combination with the $Z$-amplitude scattering will cause initially
Ion clouds $(\mathrm{z}, \mathrm{y})$ :

(a)

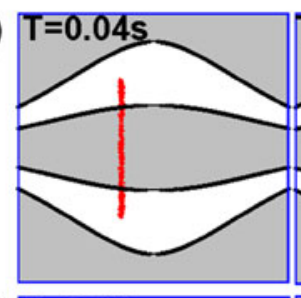

$\mathrm{T}=0.08 \mathrm{~s}$

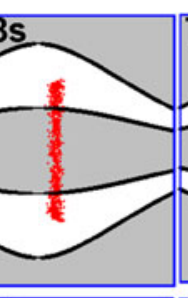

(b)

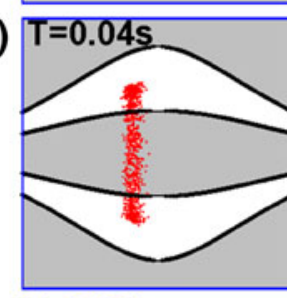

\begin{abstract}
$\mathrm{T}=0.08 \mathrm{~s}$
\end{abstract}
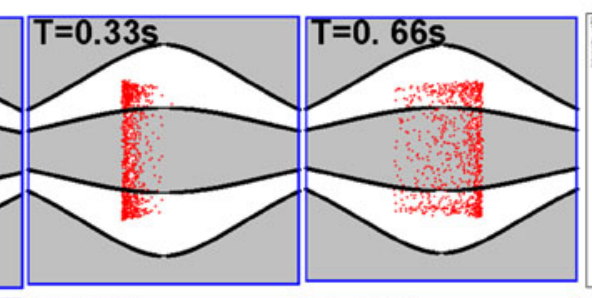

Signal(1s):

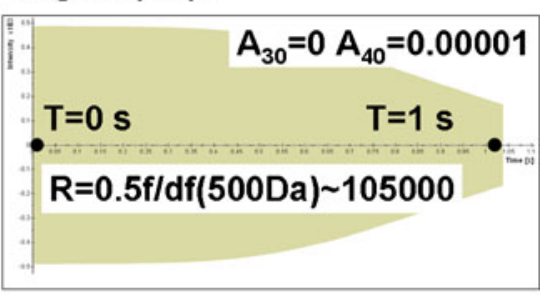

(c)

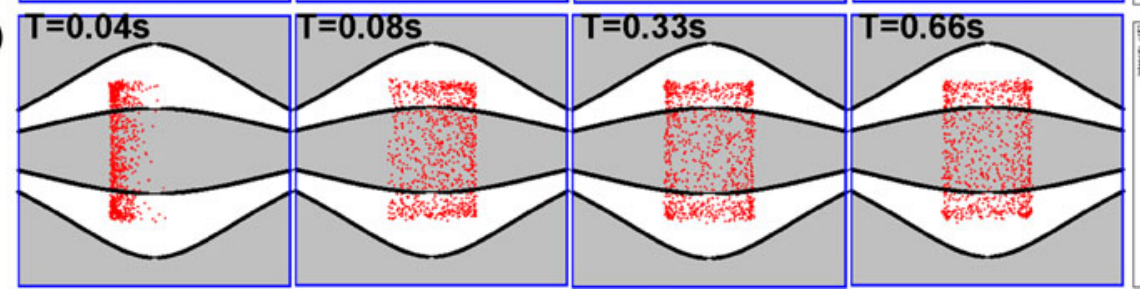

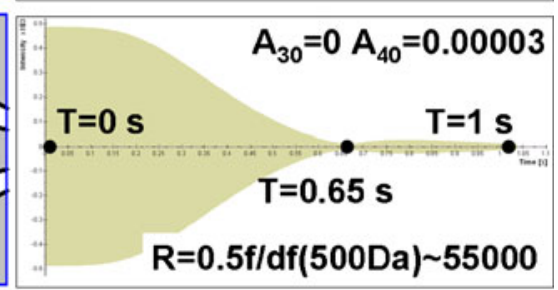

$\mathrm{T}=0.66 \mathrm{~s}$
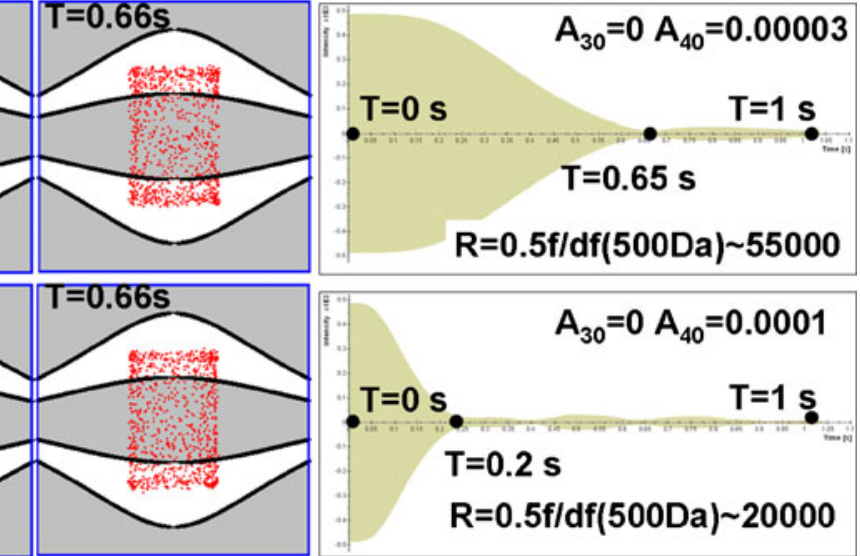

Figure 4. Evolution of ion clouds at various values of term $\frac{k A_{40}}{L^{2}}\left(8 z^{4}-24 z^{2} r^{2}+3 r^{4}\right)$ of the practical trap potential and $m / z=500, z=1 \mathrm{e}, 10^{3}$ ions. The non-harmonicity of the trapping electric potential causes decay of the signal due to ion cloud de-phasing along the $Z$ axis. Loss of phase is proportional to the term $A_{40}$ 
synchronous ion clouds to lose ions' $Z$-phase coherence in potentials of the type (15). The computer simulation that we performed let us determine the value of coefficient $A_{40}$ leading to the signal decay speed corresponding to frequency resolution $10^{5}$ at $m=500 \mathrm{Da}$ and charge state $1 \mathrm{e}$.

So far, such a field composition has been abstract due to the absence of qualitative correspondence between the expansion coefficients and measurable characteristics. To provide such a qualitative correspondence, we remember that the field is Z-symmetric and thus we perform variation of the lowest symmetric coefficient of the real field inharmonicity $-A_{40}$. Specifically, we vary it to arrive at such a decaying signal, which gives us resolution of at least $10^{5}$. Figure 4 illustrates this adjustment: assuming that the simulation region's length $L=3.81 \mathrm{~cm}$, for mass $500 \mathrm{Da}$ and charge state 1 different decays resulting in different levels of resolution $R=f / 2 \Delta f$ are produced by different values of $A_{40}: 10^{-4}, 3 \times 10^{-5}$, and $10^{-5}$. We were aiming at resolution $10^{5}$ to obtain the field approximately corresponding to the real instruments. One can see that at $A_{40}=10^{-5}$ we obtain the resolution $(R=f / 2 d f)$ slightly greater than $10^{5}$. Longer signals and greater target resolution (a)

\section{Ion clouds $(x, y),(z, y)$ :}

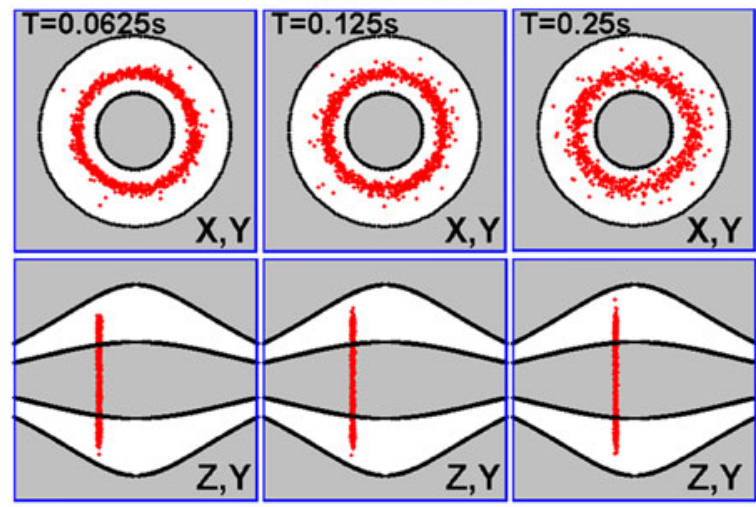

(b)

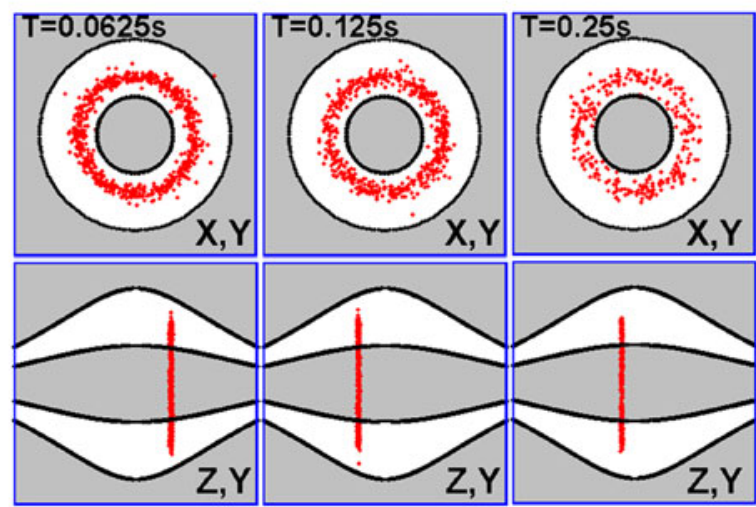

(c)

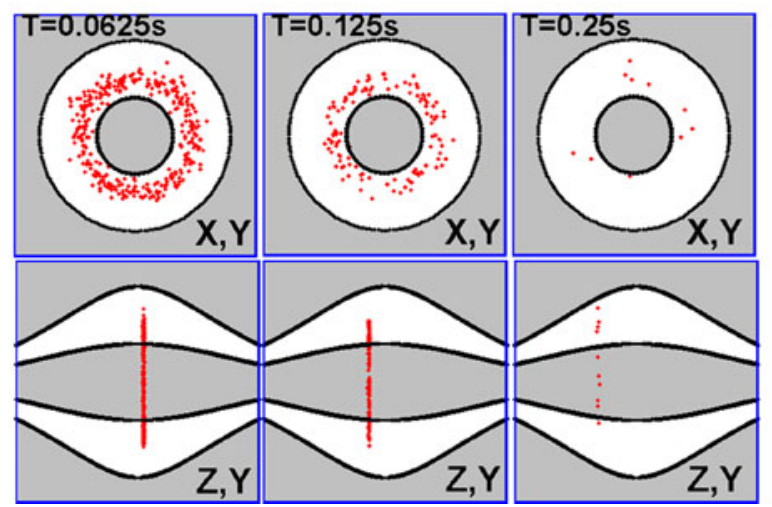

Signal(0.25s):

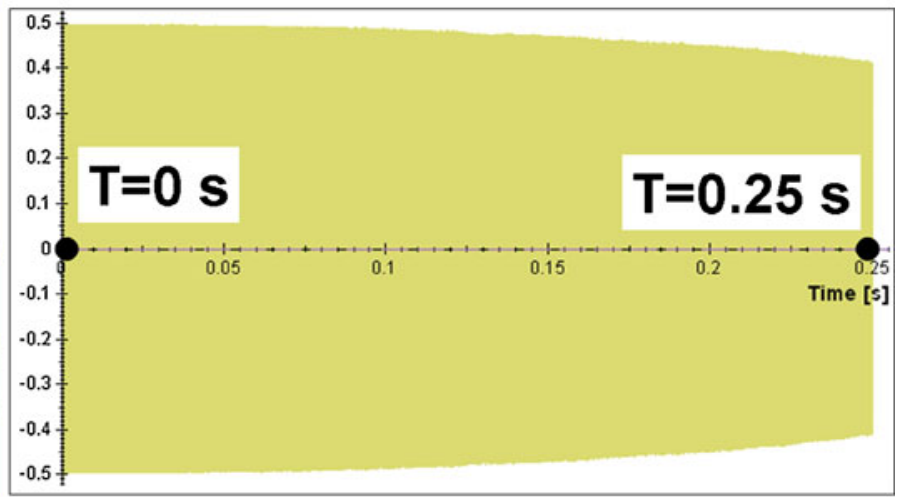

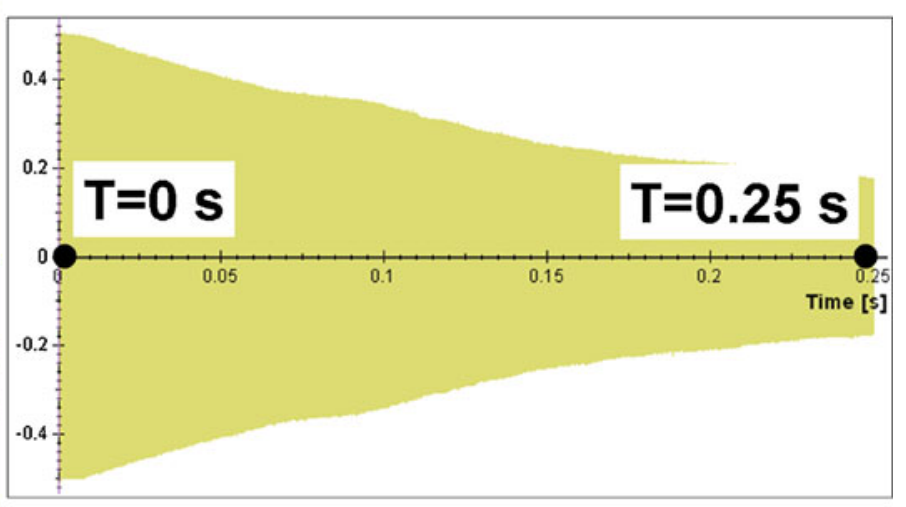

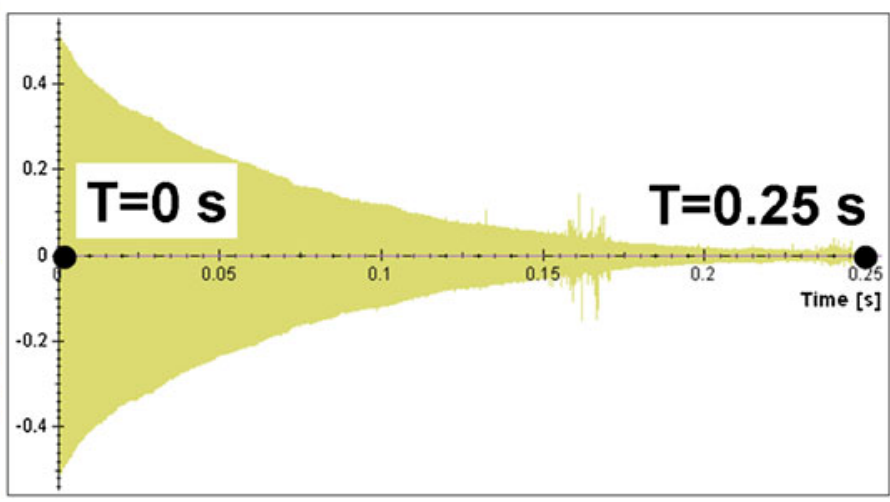

Figure 5. Cloud dynamics at various amplitudes of 20 spindle surface defects defined as deviations from the "ideal" profile $z\left(r_{1,2}\right)=\sqrt{\frac{r^{2}}{2}-\frac{R_{1,2}^{2}}{2}+R_{m}^{2} \ln \frac{R_{1,2}}{r}}$ in the $X Y$-plane. (a), (b), (c), deviation amplitudes $0.075188,0.11278$, and $0.15038 \mathrm{~mm}$, respectively 
were not available because the corresponding simulations would have exceeded available computational resource.

Additionally, the quality of machining the electrodes affects the smoothness of the electric field (7). If we represent machining defects by the maximum deviation $\delta$ of $R_{1}$ and the number of $n$ deviation segments where $k \neq$ const as a result of $R_{1}+\varepsilon_{i}$ and $\varepsilon_{i} \in[0, \delta]$, then the latter factor can be mimicked by piecewise approximation of (7) preserving the gradient of potential (see Appendix 2). Local electrostatic field inhomogeneities cause ions to deviate from their ideal orbits on a bigger "radius" leading to ion loss on the outer electrodes and increased signal decay. The result of non-smoothness of $\varphi$ on the signal is presented in Figure 5. Ellipticity of the trajectories increases with the number of deformations. As we see, the signal decay is caused by the instability in the orbital ion motion, in the regions where the ions leave the (stable) orbit. However, there is no dephasing of the ion clouds in the $Z$ direction in this case. Increased signal decay with the increase of the amplitude of deviations from the ideal surface.

\section{Frequency Shifts}

One of the factors that compromises mass measurement accuracy in the orbital trap is a systematic deviation of the observable frequency of charged particle oscillations in the axial potential gap from the theoretic frequency $\omega$ even for a single ion cloud. Therefore, improving the instrument's mass accuracy requires understanding all the processes behind such a systematic deviation in order to fix the latter by means of a calibration formula. A preliminary analysis of phase-dependent frequency shifts was done by the authors in [17].

Consider an application of the above theory to the analysis of oscillations in real i.e. finite-sized traps. In reality electrostatic potential of the type (1) cannot be created, therefore let's consider an approximation (15). The presence of new terms will significantly complicate the ion motion. Summands $\frac{A_{30} k}{L} 2 z^{3}$ and $\frac{A_{40} k}{L^{2}} 8 z^{4}$ will make $Z$-oscillations to become nonlinear and anharmonic which can be described by a motion equation

$$
\ddot{z}+\frac{q}{m} k z+\frac{q}{m} k \frac{6 A_{30}}{L} z^{2}+\frac{q}{m} k \frac{32 A_{40}}{L^{2}} z^{3}=0
$$

and solved via the method of successive approximations for the case when the contribution of term $\frac{A_{30} k}{L} 2 z^{3}$ is greater than $\frac{A_{40} k}{L^{2}} 8 z^{4}$ [18]. Thus, if we ignore the terms related to combination oscillations, we get $z=a \cos \omega t$ where $\omega=\omega_{0}\left[1+\left(\frac{12 A_{40}}{L^{2}}-\frac{15 A_{30}^{2}}{L^{2}}\right) a^{2}\right]$ and $\omega_{0}^{2}=\frac{q}{m} k$. An important property of such an anharmonic motion is a dependence of the frequency of oscillations on their amplitude which applied to the ion motion will cause frequency shifts for ions of different oscillation amplitudes. In the Figure 3 we see that $Z$-oscillation amplitudes grow with the ion population, eventually causing measurable frequency shifts.

From our simulation results for $10^{4}, 10^{5}, 10^{6}, 2 \times 10^{6}$, $3 \times 10^{6}, 4 \times 10^{6}, 5 \times 10^{6}$, and $6 \times 10^{6}$ charged particles for the standard Orbitrap geometry, frequency shifts were observed for the detection time of about $0.002 \mathrm{~s}$, which corresponds to $10^{5}$ steps of motion integration. Each step's duration was calculated the way that a single revolution around the spindle would correspond to 100 steps. Figure 6 presents the results, from which we can conclude that frequency shifts for an Orbitrap having trapping field of the form (15) can be approximated as

$$
\begin{aligned}
& f[H z]=277839-0.5 N, \text { for }_{30}=0, A_{40}=10^{-5}, L=3.81 \mathrm{~cm} \\
& f[H z]=277822-N, \text { for }_{30}=0, A_{40}=10^{-4}, L=3.81 \mathrm{~cm}
\end{aligned}
$$

This shows that the shifts can be approximated with expressions of the kind $f[\mathrm{~Hz}]=\mathrm{C}_{1}-\mathrm{C}_{2} N$, which corresponds to calibration law $m / z \approx \frac{C_{3}}{f^{2}}-\frac{C_{4} N}{f^{3}}$, while the approximated based on the experimantal data [19], gives $m / z \approx \frac{C_{5}}{f^{2}}-\frac{C_{6}}{f^{2}} \sqrt{N}$. Regarding the magnitude of shift, Gorshkov et al. ([19], Figure 1, peptide $[\mathrm{M}+2 \mathrm{H}] 2^{+}$ DNYDEGFGHR) report the frequency shift of about $4 \mathrm{ppm}$ based on the change in the AGC reading from 0 to $5 \times 10^{5}$ ions of $m / z \approx 600$. In our case, we have a linear dependency: $1 \mathrm{ppm}$ for $A_{40}=10^{-5}$ and $2 \mathrm{ppm}$ for $A_{40}=10^{-4}$ in the relative change of the measured frequency, which is quite comparable to the former up to the order of magnitude.

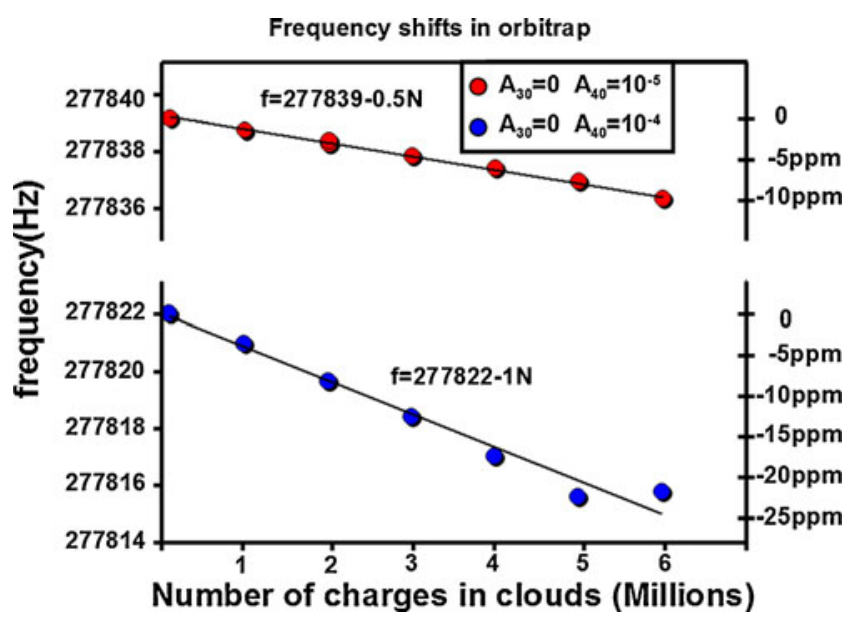

Figure 6. Space charge effect as an independent factor behind frequency shifts in Orbitrap with $R_{1}=6 \mathrm{~mm}$, $R_{2}=15 \mathrm{~mm}, R_{m} \approx R_{2} \sqrt{2}, U=3500 \mathrm{~V}, L=3.81 \mathrm{~cm}$ for $10^{4}$, $10^{5}, 10^{6}, 2 \times 10^{6}, 3 \times 10^{6}, 4 \times 10^{6}, 5 \times 10^{6}, 6 \times 10^{6}$ elementary charges in the cloud of $m / z=500, z=10 \mathrm{e}$ 


\section{Conclusion}

We present a study of the mechanism and perturbing effects of electrostatic potential inharmonicity, electrode surface irregularity, and collocation cloud population on performance of the Orbitrap mass analyzer based on the theoretical Kingdon trap potential in both ideal and finite-size geometries. The obtained theoretical results are illustrated via numerical experiments on various calibration samples and a complex ion mixture of the cytochrome $c$ isotope cluster. A series of variations of the analytical trap field were performed to test the effect of its inharmonicity and manufacturing deviations on the frequency shifts, signal quality, and dynamic range of the mass analyzer. Specifically, we characterized shifts of frequencies of axial oscillations caused by ion-ion interactions for a range of populations of ion clouds and proposed a calibration expression $m / z \approx \frac{C_{3}}{f^{2}}-\frac{C_{4} N}{f^{3}}$ as the calibration equation accounting for the shifts. Unlike in the idealized theoretical Kingdon trap geometry, to account for the signal decay caused by the ion loss, the finite axial length of the orbital trap can be adequately described by additional non-harmonic terms polynomial in the coordinate. We show that the non-ideal field of a real instrument is the biggest factor behind the loss of phase coherence in ion clouds which in turn limits the resolving power of Orbitrap based mass analyzers. We also examine another significant factor behind dephasing, which gets worse with the increase of ion population trapped in a non-ideal field. Particularly, we found that the adverse influence of shortened ends of a real instrument is adequately described by $\varphi(r, z)=\frac{k}{2}\left[\left(z^{2}-\frac{r^{2}}{2}\right)+R_{m}^{2} \ln \frac{r}{R_{m}}\right]+\frac{k A_{30}}{L}\left(2 z^{3}-3 z r^{2}\right)+\frac{k A_{40}}{L^{2}}\left(8 z^{4}-\right.$ $\left.24 z^{2} r^{2}+3 r^{4}\right)+C$. Additionally, small deviations in local field curvature cause faster decay of the signal.

\section{Acknowledgments}

The authors gratefully acknowledge Mikhail Gorshkov for his profound comments of various aspects of this work, and AMOLF's E\&I Department for technical assistance. E.N. and G.V. acknowledge the support from the Russian Foundation of Basic Research (grants 09-04-00725, 10-0413306), from the Russian Federal program (state contracts 14.740.11.0755, 16.740.11.0369), and from the Fundamental Sciences for Medicine program of the Russian Academy of Sciences.

\section{Appendix 1}

The Filter Diagonalization Method

Consider a time series $X=x_{1}, x_{2}, \ldots$ produced by some parametric process

$$
x_{n}=\sum_{k=1}^{K} d_{k} e^{-i n \tau \omega_{k}} .
$$

where $\tau$ is the time step, $n$ - step number, $d_{k}$ and $\omega_{k}-k$-th complex amplitude and frequency, respectively, and $K$ is the number of spectral elements. Two major approaches to spectrum estimation are used: non-parametric (e.g., the Fourier transform where only time series' observations are used and parametric), which is based on some assumption about the underlying process (e.g., that the series' observations were generated by some linear dynamical system like an autoregression of order $n$ ). The motivation of the latter is availability of expansions in the basis of exponentially decaying harmonics. The common idea of many parametric methods is obtaining the power spectrum of a series based on the knowledge of spectrum of a generating process' evolution operator $H$. Statistically, such an inference is consistent due to the Szego theorem. Let $R(k)=E\left[x_{i} x_{i+k}\right]$ be the autocorrelation function (ACF) of $X$ and its Fourier transform is

$$
S(\omega)=\sum_{m=-\infty}^{\infty} R(m) e^{-i m \omega},-\pi<\omega \leq \pi .
$$

Then the distribution of eigenvalues of matrix

$$
R=\left(\begin{array}{lllll}
r_{0} & r_{m-1} & \ldots & r_{2} & r_{1} \\
r_{1} & r_{0} & \ldots & r_{3} & r_{2} \\
\ldots & \ldots & \ldots & \ldots & \ldots \\
r_{m-2} & r_{m-3} & \ldots & r_{0} & r_{m-1} \\
r_{m-1} & r_{m-2} & \ldots & r_{1} & r_{0}
\end{array}\right)
$$

comprised of the ACF's elements converges to the power spectrum of $X$ as $n \rightarrow \infty$ :

$$
\lim _{n \rightarrow \infty} \frac{1}{n} \sum_{i=0}^{n-1} G(\tau)=\int_{-\frac{1}{2}}^{\frac{1}{2}} G[S(\omega)] d \omega
$$

where $G$ - a continuous function. Mandelshtam and Taylor [20] proposed to treat $X$ as an output of a linear dynamical system

$$
\Phi_{n}=H^{n} \Phi_{0}
$$

whose autocorrelation matrix is used as an approximation of its evolution operator $H$ with an unknown initial state $\Phi_{0}$, which is known to form the Krylov subspace of some linear manifold. The result is Equation (18)

$$
H^{i+1} B_{k}=h_{k} H^{i} B_{k}
$$

$B_{k}$, an eigenvector (a complex amplitude) and $h_{k}$, an eigenvalue (a complex frequency) corresponding to the generalized eigenvalue problem. By solving it we obtain the eigenvalues

$$
h_{k}=e^{-i \omega_{k} \tau}
$$

determining positions of spectrum elements, and eigenvectors $B_{k}$ determining the amplitudes and initial phases. The eigenvalue problem can be found via matrix diagonalization and, thus, in general, one can find the frequencies $\omega_{k}$. The amplitudes $d_{k}$ can then be found from (17) by solving the least- 
squares equation. However, this straightforward approach is quite expensive computationally and requires a significant amount of memory because $\operatorname{rank}\left(H_{i+1}\right)$ is determined by the length of the time series $n$ while the computational complexity of this method is $O\left(n^{3}\right)$. Apart from this problem, the spectrum produced this way contains a certain amount of noise elements. There are a number of workarounds to this issue, so let's consider the most popular one, where one avoids the global fit of calculating the Krylov basis, but rather solving the problem in multiple subsequent windows. To do this, we represent each Krylov basis function by a superposition of a small number of functions from the Fourier basis:

$$
\Psi_{j}=\sum_{k} e^{-i k \varphi_{j}} \Phi_{k}
$$

where $\Psi_{j}$ is a function in the new "local" basis. We perform it in a narrow frequency window to make the size of matrix $H^{i+1}$ in (18) significantly smaller. Note that the "local" basis $\Psi_{j}$ will contain only spectral elements from the global basis. (18) is then being solved subsequently for each window and the spectra produced are merged into the resulting spectrum. Also, the noise elements are thus evenly distributed among frequency windows and can be efficiently suppressed via the singular value decomposition procedure [21] $H^{i}=U D V^{*}$ (where $U$ and $V$ are unitary matrices and $D$ is the diagonal of ordered singular values $\lambda_{1} \lambda_{2} \ldots \lambda_{n}>0$ and $V^{*}$ is the conjugate transpose of $V$ ) with truncating diagonal elements of $D$ below a certain threshold. Programmatically, our FDM implementation is based on the guidelines by O'Connor and Aizikov [22].

\section{Appendix 2}

Simulating a Field of Deformed Central Electrode

We have $N$ deviations $z_{i}, \delta_{i}, i=\overline{(1, N)}$ of the spindle from its ideal profile

$$
z(r)_{1,2}=\sqrt{\frac{r^{2}}{2}-\frac{R_{1,2}^{2}}{2}+R_{m}^{2} \ln \frac{R_{1,2}}{r},}
$$

and the task is to find $\varphi(z, r)$ induced by the electrode having such a profile. The idealization consists in a choice of specific approximation of the profile with deviations on it to mimic the typical real-world case. Although we can calculate $\phi(z, r)$ for any profile using numerical methods, we can do that analytically by choosing a profile that provides for the agreement with the Laplace equation

$$
\nabla^{2} \varphi(z, r+\delta(z))=0 .
$$

Following the general principle of constructing spline approximators, we'll be looking for a piecewise representation of the function $r(z)$ of the spindle profile in the form

$$
r=f(z)=a+b z+c z^{2}+d z^{3}
$$

subject to common constraints:

$$
f^{\prime}\left(z_{i}\right)=f^{\prime}\left(z_{i+1}\right)
$$

and

$$
f^{\prime \prime}\left(z_{i-1}\right)=f^{\prime \prime}\left(z_{i+1}\right)
$$

given $f^{\prime}\left(z_{0}\right)=s_{0}$ and $f^{\prime}\left(z_{n-1}=s_{n}\right)$ where 0 - some initial slope on the ends, and a special constraint $\nabla \varphi(z, r)=0$ due to the fact that the profile $r(z, r+\delta(z))$ is the contour of $\varphi$ at distance $\delta \rightarrow 0$. After re-expressing $r$ in a polynomial form as

$$
r=f\left(z_{i}\right)=a_{i}+b_{i} z+c_{i} z^{2}+d_{i} z^{2}
$$

we have

$$
f_{i}^{\prime}(z)=b_{i}+2 c_{i} z+3 d_{i} z^{2}
$$

and

$$
b_{i}-b_{i+1}+2 c_{i} z-2 c_{i+1} z+3 d_{i} z^{2}-3 d_{i+1} z^{2}=0 .
$$

Accordingly,

$$
f^{\prime \prime}(z)=2 c_{i}+6 d_{i} z
$$

and

$$
2 c_{i}-2 c_{i+1}+6 d_{i} z-6 d_{i+1} z=0 .
$$

However, the interpolant should meet a certain conservation law to be used as an electrostatic potential, specifically, the Laplace equation $\nabla f^{2}=0$, which we should provide for in the form of an additional constraint. As we know, its solutions minimize the Dirichlet energy integral $\int_{A}|\nabla f|^{2} d A$, the integrated square of the gradient resulting in the smoothest possible solution over domain $A$. If we only use values from the evenly spaced $z_{0} \ldots z_{n}$ domain for fitting the interpolant, we can use a finite difference approximation of the Laplace equation via the discrete version of the meanvalue theorem for harmonic functions: denoting $f\left(z_{1}\right)$ as a central point and $f\left(z_{0}\right)$ and $f\left(z_{2}\right)$ as its left and right Cartesian neighbors, we have

$$
-\frac{f\left(z_{0}\right)}{2}+f\left(z_{1}\right)-\frac{f\left(z_{2}\right)}{2}=0 .
$$

Or, in terms of our cubic representation,

$$
\begin{aligned}
& -\frac{a_{0}+b_{0} z_{0}+c_{0} z_{0}^{2}+d_{0} z_{0}^{3}}{2}+a_{1}+b_{1} z_{1}+c_{1} z_{1}^{2}+d_{1} z_{1}^{3} \\
& -\frac{a_{2}+b_{2} z_{2}+c_{2} z_{2}^{2}+d_{2} z_{2}^{3}}{2} \\
& \quad=0 .
\end{aligned}
$$


Finally,

$$
\left(\begin{array}{cccccccccccc}
0 & 1 & 0 & 0 & 0 & 0 & 0 & 0 & 0 & 0 & 0 & 0 \\
1 & 0 & 0 & 0 & 0 & 0 & 0 & 0 & 0 & 0 & 0 & 0 \\
1 & z_{1} & z_{1}^{2} & z_{1}^{3} & 0 & 0 & 0 & 0 & 0 & 0 & 0 & 0 \\
0 & 1 & 2 z_{1} & 3 z_{1}^{2} & 0 & 0 & 0 & 0 & 0 & 0 & 0 & 0 \\
0 & 0 & 2 & 6 z_{1} & 0 & 0 & -2 & -6 z_{1} & 0 & 0 & 0 & 0 \\
0 & 0 & 0 & 0 & 1 & z_{1} & z_{1}^{2} & z_{1}^{3} & 0 & 0 & 0 & 0 \\
0 & 0 & 0 & 0 & 1 & z_{2} & z_{2}^{2} & z_{2}^{3} & 0 & 0 & 0 & 0 \\
0 & 0 & 0 & 0 & 0 & 1 & 2 z_{2} & z_{2}^{2} & 0 & -1 & -2 z_{3} & -z_{3}^{2} \\
0 & 0 & 0 & 0 & 0 & 0 & 2 & 6 z_{2} & 0 & 0 & -2 & -6 z_{3} \\
0 & 0 & 0 & 0 & 0 & 0 & 0 & 0 & 1 & z_{3} & z_{3}^{2} & z_{3}^{3} \\
0 & 0 & 0 & 0 & 0 & 0 & 0 & 0 & 1 & z_{4} & z_{4}^{2} & z_{4}^{3} \\
0 & 0 & 0 & 0 & 0 & 0 & 0 & 0 & 0 & 1 & z_{4} & z_{4}^{2} \\
\frac{-1}{2} & \frac{-1}{2 z_{0}} & \frac{-1}{2 z_{0}^{2}} & \frac{-1}{2 z_{0}^{3}} & 1 & z_{1} & z_{1}^{2} & z_{1}^{3} & \frac{-1}{2} & \frac{-1}{2 z_{2}} & \frac{-1}{2 z_{2}^{2}} & \frac{-1}{2 z_{2}^{3}}
\end{array}\right)\left(\begin{array}{c}
a_{0} \\
b_{0} \\
c_{0} \\
d_{0} \\
a_{1} \\
b_{1} \\
c_{1} \\
d_{1} \\
a_{2} \\
b_{2} \\
c_{2} \\
d_{2}
\end{array}\right)=\left(\begin{array}{c}
y^{\prime}\left(z_{0}\right) \\
y\left(z_{0}\right) \\
y\left(z_{1}\right) \\
0 \\
0 \\
y\left(z_{1}\right) \\
y\left(z_{2}\right) \\
0 \\
0 \\
y\left(z_{3}\right) \\
y\left(z_{4}\right) \\
y^{\prime}\left(z_{4}\right) \\
0
\end{array}\right)
$$

or

$$
A x=y .
$$

The system of equations is over-determined, requiring a least-squares solution for the best fit of $A$ minimizing the Euclidian norm of the error $\|A x-y\|_{2}$. Note that the resulting spline will cause the set of points specified as initial deviation values to be adjusted to reflect the required compliance with the Laplacian regularizer. The MATLAB implementation of the singular value decomposition method was used for this.

\section{References}

1. Marshall, A.G., Hendrickson, C.L., Jackson, G.S.: Fourier transform ion cyclotron resonance mass spectrometry: A primer. Mass Spectrom. Rev. 17, 1-35 (1998)

2. Boldin, I.A., Nikolaev, E.N.: Fourier transform ion cyclotron resonance cell with dynamic harmonization of the electric field in the whole volume by shaping of the excitation and detection electrode assembly. Rapid Commun. Mass Spectrom. 25, 122-126 (2011)

3. Nikolaev, E.N., Boldin, I.A., Jertz, R., Baykut, G.: Initial experimental characterization of a new ultra-high resolution FT-ICR Cell with dynamic harmonization. J. Am. Soc. Mass Spectrom. 22, 1125-1129 (2011)

4. Kingdon, K.H.: A method for the neutralization of electron space charge by positive ionization at very low gas pressures. Phys. Rev. 21(4), 408418 (1923)

5. Lange, O., Horning, S., Makarov, A., Denisov, E.: Dynamic range of mass accuracy in LTQ Orbitrap hybrid mass spectrometer. J. Am. Soc. Mass Spectrom. 17, 977-982 (2006)

6. Perry, R., Cooks, G., Noll, R.: Orbitrap mass spectrometry: Instrumentation, ion motion and applications. Mass Spectrom. Rev. 27(6), 661699 (2008)

7. Makarov, A., Denisov, E., Kholomeev, A., Balschun, W., Lange, O., Strupat, K., Horning, S.: Performance evaluation of a hybrid linear ion trap/Orbitrap mass spectrometer. Anal. Chem. 78, 2113-2120 (2006)

8. Makarov, A.: Electrostatic axially harmonic Orbital trapping: a highperformance technique of mass analysis. Anal. Chem. 72, 1156-62 (2000)

9. Lewis, R.R.: Motion of ions in the Kingdon trap. J. Appl. Phys. 53, 3975-3980 (1982)

10. Nikolaev, E.N., Heeren, R.M.A., Popov, A.M., Pozdneev, A.V., Chingin, K.S.: Realistic modeling of ion cloud motion in a Fourier transform ion cyclotron resonance cell by use of a particle-in-cell approach. Rapid Commun. Mass Spectrom. 21, 3527-3546 (2007)

11. Makarov, A., Denisov, E., Lange, O.: Performance eEvaluation of a high-field Orbitrap mass analyzer. J. Am. Soc. Mass Spectrom. 20, 1391-1396 (2009)

12. Hardman, M., Makarov, A.: Interfacing the Orbitrap mass analyzer to an electrospray ion source. Anal. Chem. 75, 1699-1705 (2003)

13. Hu, Q., Noll, R.J., Li, H., Makarov, A., Hardman, M., Cooks, R.G.: The Orbitrap: a new mass spectrometer. J. Mass Spectrom. 40, 430-443 (2005)

14. Monroe, M. Molecular Weight Calculator (http://omics.pnl.gov/software/ MWCalculator.php), Version 6.48, October 10, 2010, accessed

15. Marshall, A.G., Grosshans, P.B.: Fourier transform ion cyclotron resonance mass spectrometry: the teenage years. Anal. Chem. 63(4), 215A- 229A (1991)

16. Jackson, J. D. (1998). Classical Electrodynamics, 3rd ed., New York: John Wiley \& Sons, ISBN 978-0-471-30932-1, sec. 3.5.

17. Leach, F. E. III, Kharchenko, A., Vladimirov, G., Aizikov, K., O’Connor, P. B., Nikolaev, E., Heeren, R. M. A., Amster, I. J.: Analysis of phase-dependent frequency shifts in simulated FTMS transients using the filter diagonalization method. J. Am. Soc. Mass Spectrom (2011) unpublished, (submitted)

18. Landau, L.D., Lifshitz E.M.: Mechanics. Vol. 1 3rd ed. ButterworthHeinemann: (1976) ISBN 978-0-750-62896-9.

19. Gorshkov, M.V., Good, D.M., Lyutvinskiy, Y., Yang, H., Zubarev, R. A.: Calibration function for the Orbitrap FTMS accounting for the space charge effect. J. Am. Soc. Mass Spectrom. 21(11), 1846-1851 (2010)

20. Mandelshtam, V.A., Taylor, H.S.: Harmonic inversion of time signals. J. Chem. Phys. 107(17), 6756-6769 (1997)

21. Forsythe, G. E., Malcolm, M. A., Moler, C. B.: Computer Methods for Mathematical Computations. Prentice Hall: Englewood Cliffs, New Jersey, 1977

22. Aizikov, K., O'Connor, P.B.: Use of the filter diagonalization method in the study of space charge related frequency modulation in Fourier transform ion cyclotron resonance mass spectrometry. J. Am. Soc. Mass Spectrom. 17, 836-843 (2006) 